\title{
Fiber transport of spatially entangled photons
}

\author{
W. Löffler, ${ }^{1, *}$ T. G. Euser, ${ }^{2}$ E. R. Eliel, ${ }^{1}$ M. Scharrer, ${ }^{2}$ P. St.J. Russell, ${ }^{2}$ and J. P. Woerdman ${ }^{1}$ \\ ${ }^{1}$ Huygens Laboratory, Leiden University, P.O. Box 9504, 2300 RA Leiden, The Netherlands \\ ${ }^{2}$ Max Planck Institute for the Science of Light, Günther-Scharowsky-Str. 1, 91058 Erlangen, Germany
}

Entanglement in the spatial degrees of freedom of photons is an interesting resource for quantum information. For practical distribution of such entangled photons it is desireable to use an optical fiber, which in this case has to support multiple transverse modes. Here we report the use of a hollow-core photonic crystal fiber to transport spatially entangled qubits.

PACS numbers:

The non-classical correlations of two entangled photons enable quantum communication and cryptography. Traditionally, polarization entanglement is utilized; however, polarization is a two-dimensional degree of freedom only (qubit). Higher-dimensional entangled systems show promise [1, 2], because more entangled degrees of freedom per particle imply stronger non-classical correlations. Examples are the violation of a Bell-type inequality [3, 4] and demonstration of the quantum coin tossing protocol [5]. The photon's temporal, frequency and spatial degrees of freedom give access to such highdimensional entanglement; enabling temporal entanglement [4, 6, 7, frequency entanglement [8, 9], and spatial entanglement [3, 10 16], respectively. These degrees of freedom are intrinsically continuous; after appropriate discretization, qudits of arbitrary dimension $d$ can be defined. Another approach for multi-dimensional entanglement is the combination of different degrees of freedom [17. 20].

Optical-fiber transport of entangled photons allows new possibilities in applied quantum information; so far this has been realized for photons entangled in polarization [21, time 22] and frequency [8, 9, but not for spatial entanglement. To transport spatially entangled photons through a fiber, this must obviously support multiple transverse modes. However, this presents a challenge since conventional multimode fibers suffer from strong intermodal coupling which tends to destroy the fragile quantum correlations carried by the spatially entangled state. Often this happens within a few $\mathrm{mm}$ of propagation 23 39]. It is thus imperative to choose a type of fiber where these decohering effects are minimal or absent, i.e., to be as close as possible to free-space propagation. Therefore, the natural choice for fiber transport of spatially entangled photons is to use a hollow-core fiber, where the light is guided essentially in air (see inset Fig. $1 b)$. Here we demonstrate that this approach is successful.

We use a hollow-core photonic crystal fiber (HC-PCF) [24, 25] with a kagomé-style cladding [26]28], see Fig. 1b. The cladding lattice is formed from sub- $\mu \mathrm{m}$ thick fused silica glass membranes, the central hollow core being created by removing a few unit cells. Our HC-PCF has a relatively large core diameter, namely $25 \mu \mathrm{m}$, and does not support bound modes because the cladding does not pos-

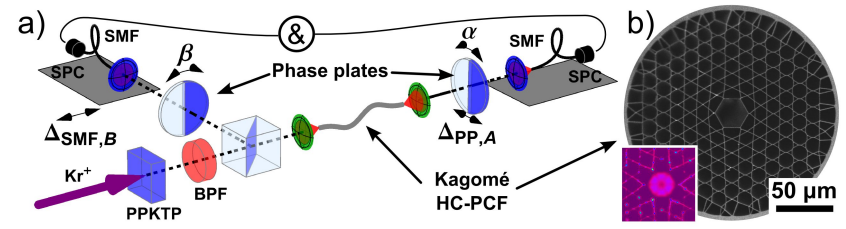

Figure 1: (Color online) (a) Experimental setup. (b) Electron micrograph of the cross-section of the HC-PCF. The core diameter is $25 \mu \mathrm{m}$, which results in an half opening angle of the fundamental mode of $44 \mathrm{mrad}$. The inset shows a near-field optical image (same scale) of the core area, acquired using incoherent $(800 \pm 10 \mathrm{~nm})$ illumination, this shows approximately circular mode confinement.

sess a photonic band gap and the negative index difference between core and cladding rules out the possibility of total internal reflection. Many Mie-like resonances exist in the core, each with a different axial wavevector component. In some respects these modes are similar to the leaky modes of hollow cylindrical capillary waveguides [29], with an important difference: with appropriate design the fundamental mode of the HC-PCF can be guided with losses as much as 100 times lower than in a capillary of the same diameter 27. It has a transverse intensity profile that approximately follows a squared $J_{0}$ Bessel function, its first zero coinciding with the core boundary. The losses are larger for higher-order modes and this limits effectively the number of propagating modes. In the experiments reported here, 3 modes are present after propagation along $30 \mathrm{~cm}$ of our kagomé HC-PCF. These modes have large overlap with the 3 lowest-order free-space paraxial Hermite-Gaussian modes (or superpositions thereof). In the Hermite-Gauss basis $H G_{m, n}$ with the polynomial index $m$ and $n$ in $x$ and $y$, these are the $H G_{0,0}, H G_{1,0}$, and $H G_{0,1}$ modes. We find experimentally that the $30 \mathrm{~cm}$ kagome fiber attenuates the first order modes by $\approx 8 \%$ compared to the fundamental mode, this agrees well with $0.7 \mathrm{~dB} / \mathrm{m}$ or $5 \%$ as quoted in 28] for a similar fiber.

In our experiments we study the effect of fiber transport on quantum correlations between two entangled qubits, where spatially entangled photons are produced by spontaneous parametric downconversion (SPDC, type-I, collinear phase matching) [11. The setup 
is sketched in Fig. 1: The entangled two-photon state is generated in a $2 \mathrm{~mm}$ long periodically-poled KTP crystal by pumping it with a weakly focused $\left(w_{0}=250 \mu \mathrm{m}\right)$ $413 \mathrm{~nm} \mathrm{Kr}{ }^{+}$laser beam $(80 \mathrm{~mW})$. The polarization state of the photons is not relevant here since polarization and spatial degrees of freedom are decoupled (paraxial regime). The two entangled photons are filtered spectrally (BPF, $1 \mathrm{~nm}$ bandwidth, centered at $826.1 \mathrm{~nm}$ ), then the photons are probabilistically separated by a beam splitter, and one of them (path A) is transported through the $30 \mathrm{~cm}$ long HC-PCF by appropriately modematched in- and out-coupling (10x, 0.25 NA objectives). For our goal it is sufficient that only one photon passes through the HC-PCF. No special care was taken to keep the fiber straight; typically curves with a bending radius of $30 \mathrm{~cm}$ occurred. Both photons from a pair are then projected onto separate superpositions. This is done by first sending the photons through a step phase plate 30; this plate acts as a mode converter; it shifts the optical phase by $\pi$ in one half of the transverse plane with respect to the other half. Subsequently, we project this mode onto the fundamental Gaussian mode by coupling into a standard single-mode fiber. This fiber is connected to a single-photon counter and correlated photon pairs are post-selected by coincidence detection (2 ns timewindow). The entangled two-photon state as produced by SPDC [11, 30, 31] is filtered by the "3-mode" HCPCF such that we deal effectively with a $3 \mathrm{D}$ spatially entangled state formed by the 3 lowest-order Gaussian modes [3, 12]. The single-photon basis states for these modes are $\left\{\left|H G_{0,0}\right\rangle,\left|H G_{1,0}\right\rangle,\left|H G_{0,1}\right\rangle\right\}$ in the HermiteGauss basis. Such a bipartite entangled state lives in a $\mathbb{C}^{3} \otimes \mathbb{C}^{3}$ Hilbert space which can be explored by investigating spatial correlations in two non-identical but partly overlapping $\mathbb{C}^{2} \otimes \mathbb{C}^{2}$ subspaces.

As a first step, we consider the subspace spanned by the degenerate $\left|H G_{1,0}\right\rangle$ and $\left|H G_{0,1}\right\rangle$ modes. We center both phase plate projectors to the single-mode detection fibers $\left(\Delta_{S M F, B}=\Delta_{P P, A}=0\right.$, see Fig. 1). In the corresponding 2D Hilbert subspace, each detector projects on the superposition state $\sin (\phi)\left|H G_{1,0}\right\rangle+\cos (\phi)\left|H G_{0,1}\right\rangle$, which depends on the orientation $\phi$ of the phase plate ( $=\alpha$ or $\beta$ for phase plate A or B, respectively, see Fig. 1a). This experiment is analogous to the $2 \mathrm{D}$ polarizationentanglement case [32, 33] and thus, the resulting coincidence fringes are sinusoidal (Fig. 2). We also see anisotropic effects of the fiber: the visibility of the diagonal $\left( \pm 45^{\circ}\right)$ fringes is reduced compared to that of the $\left(0^{\circ}, 90^{\circ}\right.$ degrees $)$ fringes. Also, mode rotation occurs as the fringes are slightly dephased with respect to the relative orientation $(\alpha-\beta)$ of the phase plates. We attribute these effects to the broken rotational symmetry in the fiber: the cladding has a hexagonal symmetry; this weakly affects the modes since a small fraction of the optical field resides in the cladding. This leads to mode mixing and mode rotation; both could be avoided if one were to use a circular-core concentric Bragg fiber [34].

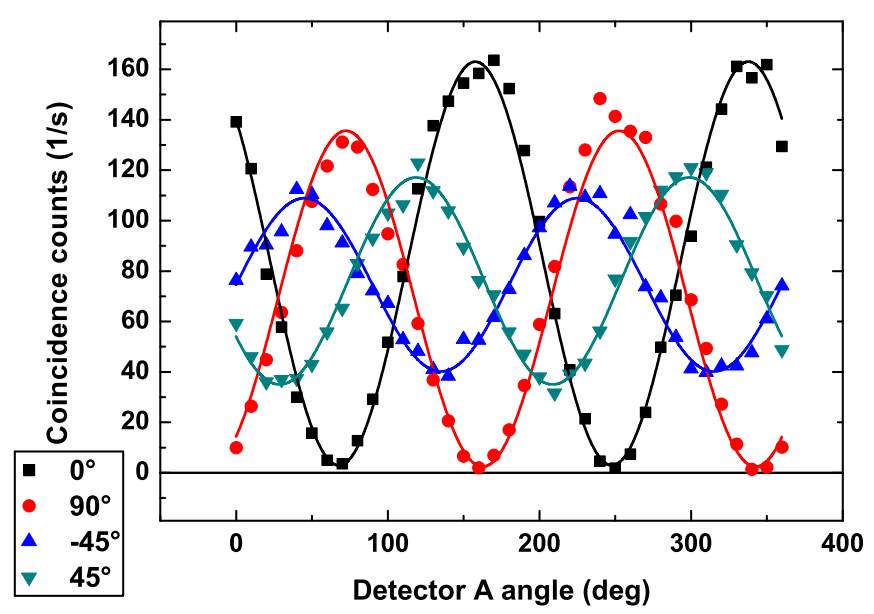

Figure 2: (Color online) Fiber transport of 2D spatially entangled photons in the degenerate case (i.e. superpositions of the $\left|H G_{1,0}\right\rangle$ and $\left|H G_{0,1}\right\rangle$ states). Both mode analyzers are equipped with a step phase plate which can be rotated. To obtain a coincidence fringe, plate $\mathrm{A}$ is rotated, while plate $\mathrm{B}$ is kept fixed. No significant fringe is detected in the singledetector count rates.

In polarization-based Bell experiments, maximum violation happens for, e.g., $\left(\alpha_{1}, \alpha_{2}, \beta_{1}, \beta_{2}\right)=$ $\left(0^{\circ}, 45^{\circ}, 22.5^{\circ}, 67.5^{\circ}\right)$. In our case, we expect these angles to be different due to the detrimental effects mentioned above. In order to find the maximum Bell-violation angles, we plot the CHSH S-parameter 35. for $\left(\alpha_{1}, \alpha_{2}\right)=\left(0^{\circ}, 45^{\circ}\right)$ as a function of $\left(\beta_{1}, \beta_{2}\right)$ (see Fig. 3). Each pixel shows color-coded the value of the S-parameter for one set of angles; regions with $S>2$ are colored white. The overall pattern of Fig. 3 is very similar to the ideal case; but the peaks are shifted. A numerically search for the maximum Bell violation results in the angles $\left(\alpha_{1}, \alpha_{2}, \beta_{1}, \beta_{2}\right)=\left(0^{\circ},-45^{\circ}, 270^{\circ}, 150^{\circ}\right)$, where $S=2.17 \pm 0.04$. The uncertainty (standard deviation) of $S$ is calculated from the uncertainty $\Delta N$ in the coincidence counts $N$ using Gaussian propagation of uncertainty. For this 2D subspace we violate the Bell inequality by 4 standard deviations; thus proving that spatial entanglement of the degenerate $\left|H G_{1,0}\right\rangle$ and $\left|H G_{0,1}\right\rangle$ states survives fiber propagation.

As the second step, we study the 2D subspace spanned by the non-degenerate states $\left|H G_{0,0}\right\rangle$ and $\left|H G_{1,0}\right\rangle$. In this case it is necessary to consider first the intermodal dispersion of the relevant modes in the fiber, i.e., the relative propagation constants of the $H G_{0,0}$ and $H G_{1,0}$ modes. We measure this by launching a well-known (classical) coherent superposition of these modes into the fiber and observe, as a function of wavelength, the near-field intensity at the fiber exit. From the mode beating we determine the intermodal dispersion 36 to be $1.5 \mathrm{ps} / \mathrm{m}$. This agrees well with the calculated value of $1.6 \mathrm{ps} / \mathrm{m}$ obtained by approximating the HC-PCF by a hollow dielectric capillary [29] (with $r=12.5 \mu \mathrm{m}, \lambda=826 \mathrm{~nm}$, and $n=1.45$ ) [40]. Although this intermodal dispersion 


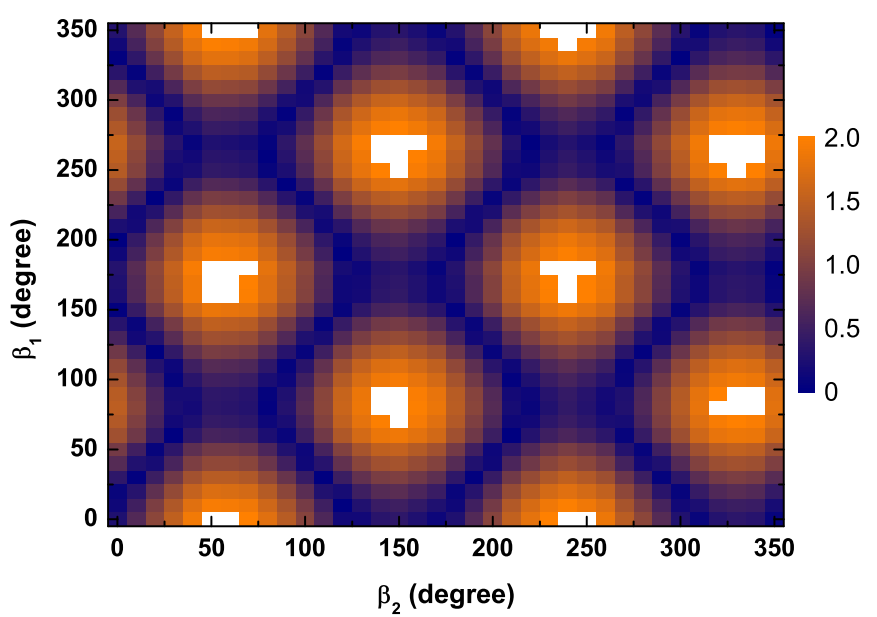

Figure 3: Parameter scan of the CHSH S-parameter as a function of $\left(\beta_{1}, \beta_{2}\right)$ for fixed $\left(\alpha_{1}, \alpha_{2}\right)$. Each pixel shows colorcoded $S\left(\alpha_{1}=0^{\circ}, \alpha_{2}=-45^{\circ}, \beta_{1}, \beta_{2}\right)$, which is calculated using 16 different coincidence count measurements at different orientations of the phase plates. Regions where the CHSH inequality $(S \leq 2)$ is violated are colored white.

is small, we need to limit the bandwidth of the downconverted photons, in order to preserve the coherence of the non-degenerate quantum state; we use 1-nm bandwidth filters in the experiments with the $30-\mathrm{cm}$ long HCPCF.

In this non-degenerate case we have performed a quantum interference experiment somewhat similar to that of Mair et al. [11]: In analyzer A, we no longer center the phase plate but give it a transverse (i.e. in-plane) offset $\Delta_{P P, A}$ with respect to the single-mode fiber (Fig. 1a). This detector configuration projects the incoming photon onto a superposition state $a_{0}\left|H G_{0,0}\right\rangle+a_{1}\left|H G_{1,0}\right\rangle$. The intensity distribution corresponding to this superposition carries a nodal line. This is probed non-locally by using analyzer B as a Gaussian probe, i.e. we remove its phase plate. For a certain offset $\Delta_{P P, A}$, we scan the fundamental-mode analyzer B $\left(\Delta_{S M F, B}\right)$ transversely to the optical axis and normal to the edge of the phase plate of analyzer A. We expect that the coincidence fringe resembles the coherent superposition onto which photon $\mathrm{A}$ is projected, and that the dip in this fringe moves proportionally to the phase plate A offset $\Delta_{P P, A}$. This nonlocal shift is clearly visible in our experimental results shown in Fig. 4: We observe a dip in the coincidence counts (normalized to the single counts) at twice the phase step offset $\Delta_{P P, A}$, in agreement with theory (the beam waist at the phase plates is $w_{0}=0.8 \mathrm{~mm}$ ). Considering that the theory does not include mode-dependent attenuation or intermodal dispersion of the $H G_{0,0}$ and $H G_{1,0}$ modes in the fiber, the overall correspondence with the experimental data is good. This demonstration of nonlocality is obviously not equivalent to a Bell test of entanglement, but can be seen as a first step in that direction.

Finally, we discuss briefly the key issues in fiber trans-

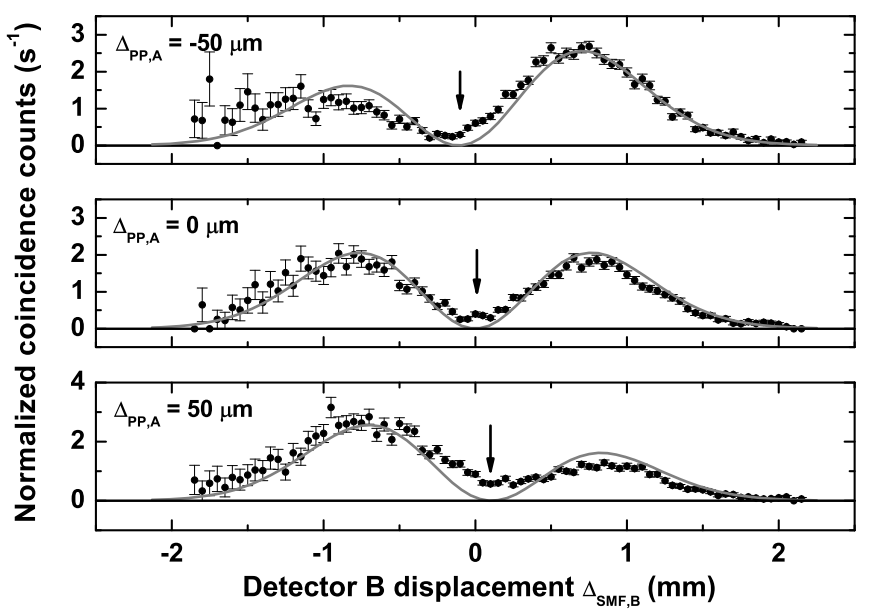

Figure 4: Fiber transport of a non-degenerate superposition of photons modes. Analyzer A projects on a superposition of $\left|H G_{0,0}\right\rangle$ and $\left|H G_{1,0}\right\rangle$, analyzer B (Gaussian probe) scans normal to the phase plate step of analyzer A. The dip in coincidence counts (arrow), is directly proportional to the phase plate displacement $\Delta_{P P, A}$. The experimental coincidence count rate is normalized to the single-detector count rate, and the theoretical curves (through lines) are scaled vertically with the same constant to match the data. The visibility of the three curves is $76 \%, 74 \%$ and $55 \%$ (from top to bottom). We define the visibility here as (MAX-MIN)/(MAX + MIN) where MAX is the average of the count rates in the two peaks that are adjacent to the dip, and MIN is the count rate in the dip.

port of multi-mode quantum superpositions: intermodal dispersion and intermodal mixing. Intermodal dispersion leads to decoherence of the quantum superposition during fiber transport, this must be small compared to the photon bandwidth. Therefore, the spread in the transverse wavevector $k_{\perp}$ of the modes should be small. This is the case for weakly confining fibers with a large core and consequently large mode area (compared to the wavelength). Additionally, one could employ a special subset of the modes with nearly equal propagation constants; for large-core fibers (with negligible diffraction corrections) these are the modes within one mode order $m+n$ in the case of $H G_{m, n}$ modes [37. However, the future applicability of fiber transport of spatially entangled photons will be ultimately limited by unavoidable fabrication tolerances as well as externally applied strain, stress and bends; this does eventually lead to intermodal mixing. Due to geometric reasons a large-diameter mode, as appearing in large-core fibers, is less sensitive to perturbations. Since perturbations act via local refractiveindex changes, it is advantageous, as stressed in the introduction, to use a hollow-core fiber to transport the light essentially in air, so as to minimize these effects. Since index-guidance is impossible in hollow-core fibers, guidance in such fibers must be preferably provided by a photonic bandgap of the cladding. Our kagomé-lattice fiber does not provide such lossless guiding and is there- 
fore not ideal; mode dependent losses may lead to nonorthogonality of the modes and thus to mode mixing [38]. It is encouraging that this potential problem has not prevented the experiments reported in this Letter.

In conclusion, we have shown the first experimental demonstration of the transport of spatially entangled photons through an optical fiber. In the degenerate subspace of the $\left|H G_{1,0}\right\rangle$ and $\left|H G_{0,1}\right\rangle$ states, we demonstrated entanglement of the fiber-transported spatial qubit by Bell inequality violation. Although this entanglement is demonstrated only in the context of a degenerate qubit Hilbert space, given the known and previously demonstrated potential of spatial modes to provide higher-dimensional entanglement $(d>2)$ [3, 11-13] our results can be regarded as a proof-of-principle that the transport of higher-dimensional $(d>2)$ spatially entangled photons through fibers might be possible in future. Our demonstration of quantum interference in a nondegenerate 2D Hilbert space (Fig. 4) is a first step in this direction.

We acknowledge fruitful discussions with M. P. van Exter and G. Nienhuis and financial support by NWO and the EU FET-Open grant HIDEAS (FP7-ICT- 221906).

* Electronic address: loeffler@physics.leidenuniv.nl

[1] D. Collins, N. Gisin, N. Linden, S. Massar, and S. Popescu, Phys. Rev. Lett., 88, 040404 (2002).

[2] M. Fujiwara, M. Takeoka, J. Mizuno, and M. Sasaki, Phys. Rev. Lett., 90, 167906 (2003).

[3] A. Vaziri, G. Weihs, and A. Zeilinger, Phys. Rev. Lett., 89, 240401 (2002)

[4] R. T. Thew, A. Acín, H. Zbinden, and N. Gisin, Phys. Rev. Lett., 93, 010503 (2004).

[5] G. Molina-Terriza, A. Vaziri, R. Ursin, and A. Zeilinger, Phys. Rev. Lett., 94, 040501 (2005).

[6] J. D. Franson, Phys. Rev. Lett., 62, 2205 (1989).

[7] H. D. Riedmatten, I. Marcikic, H. Zbinden, and N. Gisin, Quant. Inf. Comp., 2, 425 (2002).

[8] S. Ramelow, L. Ratschbacher, A. Fedrizzi, N. K. Langford, and A. Zeilinger, Phys. Rev. Lett., 103, 253601 (2009).

[9] L. Olislager, J. Cussey, A. T. Nguyen, P. Emplit, S. Massar, J.-M. Merolla, and K. P. Huy, Phys. Rev. A, 82, 013804 (2010).

[10] J. G. Rarity and P. R. Tapster, Phys. Rev. Lett., 64, 2495 (1990).

[11] A. Mair, A. Vaziri, G. Weihs, and A. Zeilinger, Nature, 412, 313 (2001)

[12] N. K. Langford, R. B. Dalton, M. D. Harvey, J. L. O'Brien, G. J. Pryde, A. Gilchrist, S. D. Bartlett, and A. G. White, Phys. Rev. Lett., 93, 053601 (2004).

[13] M. N. O'Sullivan-Hale, I. Ali Khan, R. W. Boyd, and J. C. Howell, Phys. Rev. Lett., 94, 220501 (2005).

[14] S. S. R. Oemrawsingh, X. Ma, D. Voigt, A. Aiello, E. R. Eliel, G. W. 't Hooft, and J. P. Woerdman, Phys. Rev. Lett., 95, 240501 (2005).

[15] B. Jack, J. Leach, J. Romero, S. Franke-Arnold, M. Ritsch-Marte, S. M. Barnett, and M. J. Padgett,
Phys. Rev. Lett., 103, 083602 (2009).

[16] G. Taguchi, T. Dougakiuchi, M. Iinuma, H. F. Hofmann, and Y. Kadoya, Phys. Rev. A, 80, 062102 (2009).

[17] D. V. Strekalov, T. B. Pittman, A. V. Sergienko, Y. H. Shih, and P. G. Kwiat, Phys. Rev. A, 54, R1 (1996).

[18] J. T. Barreiro, N. K. Langford, N. A. Peters, and P. G. Kwiat, Phys. Rev. Lett., 95, 260501 (2005).

[19] T. Yang, Q. Zhang, J. Zhang, J. Yin, Z. Zhao, M. Żukowski, Z.-B. Chen, and J.-W. Pan, Phys. Rev. Lett., 95, 240406 (2005).

[20] C. Cinelli, M. Barbieri, R. Perris, P. Mataloni, and F. D. Martini, Phys. Rev. Lett., 95, 240405 (2005).

[21] A. Poppe, A. Fedrizzi, R. Ursin, H. Böhm, T. Lörunser, O. Maurhardt, M. Peev, M. Suda, C. Kurtsiefer, H. Weinfurter, T. Jennewein, and A. Zeilinger, Opt. Express, 12, 3865 (2004)

[22] I. Marcikic, H. de Riedmatten, W. Tittel, H. Zbinden, M. Legré, and N. Gisin, Phys. Rev. Lett., 93, 180502 (2004).

[23] A. Lucesoli and T. Rozzi, Appl. Opt., 46, 3031 (2007).

[24] J. C. Knight, J. Broeng, T. A. Birks, and P. S. J. Russell, Science, 282, 1476 (1998).

[25] O. Shapira, A. F. Abouraddy, Q. Hu, D. Shemuly, J. D. Joannopoulos, and Y. Fink, Opt. Express, 18, 12622 (2010).

[26] F. Benabid, J. C. Knight, G. Antonopoulos, and P. S. J. Russell, Science, 298, 399 (2002).

[27] F. Couny, F. Benabid, and P. S. Light, Opt. Lett., 31, 3574 (2006).

[28] T. G. Euser, G. Whyte, M. Scharrer, J. S. Y. Chen, A. Abdolvand, J. Nold, C. F. Kaminski, and P. S. J. Russell, Opt. Express, 16, 17972 (2008).

[29] E. A. J. Marcatili and R. A. Schmeltzer, Bell Syst. Tech. J., 43, 1783 (1964).

[30] J. B. Pors, S. S. R. Oemrawsingh, A. Aiello, M. P. van Exter, E. R. Eliel, G. W. 't Hooft, and J. P. Woerdman, Phys. Rev. Lett., 101, 120502 (2008).

[31] S. P. Walborn, S. Pádua, and C. H. Monken, Phys. Rev. A, 71, 053812 (2005).

[32] P. G. Kwiat, K. Mattle, H. Weinfurter, A. Zeilinger, A. V. Sergienko, and Y. Shih, Phys. Rev. Lett., 75, 4337 (1995).

[33] M. J. Padgett and J. Courtial, Opt. Lett., 24, 430 (1999).

[34] S. Johnson, M. Ibanescu, M. Skorobogatiy, O. Weisberg, T. Engeness, M. Soljacic, S. Jacobs, J. Joannopoulos, and Y. Fink, Opt. Express, 9, 748 (2001).

[35] J. F. Clauser, M. A. Horne, A. Shimony, and R. A. Holt, Phys. Rev. Lett., 23, 880 (1969).

[36] P. Hlubina, J. Mod. Opt., 42, 2385 (1995).

[37] Y. Ma, Y. Sych, G. Onishchukov, S. Ramachandran, U. Peschel, B. Schmauss, and G. Leuchs, Appl. Phys. B, 96, 345 (2009).

[38] A. E. Siegman, Phys. Rev. A, 39, 1253 (1989).

[39] Also our own experiments with conventional multi-mode fibers show decoherence after a few millimeters.

[40] The intermodal dispersion of our kagomé HC-PCF is accordingly much lower than that of conventional indexguided multi-mode fibers (around $50 \mathrm{ps} / \mathrm{m}$ ) 